\title{
Algoritmo de Roteamento Assistido para Comunicação D2D em Sistemas de Segurança Pública sobre Redes LTE Avançadas
}

\author{
Alex Vidigal Bastos ${ }^{1,2}$, Cristiano M. Silva ${ }^{1}$, Diógenes Cecílio da Silva Júnior ${ }^{2}$ \\ ${ }^{1}$ Universidade Federal de São João Del-rei (UFSJ) - MG - Brasil \\ ${ }^{2}$ Programa de Pós-Graduação em Engenharia Elétrica \\ Universidade Federal de Minas Gerais (UFMG) \\ Av. Antônio Carlos 6627, 31270-901, Belo Horizonte, MG, Brasil \\ \{alexvbh, cristiano\}@ufsj.edu.br, diogenes@ufmg.br
}

\begin{abstract}
This paper describes a routing algorithm for device-to-device (D2D) communication in cellular architectures with the goal to extend the coverage of base stations in public safety scenarios. The proposed algorithm takes into account that D2D communications in cellular networks are managed by base stations. The Load Balancing Based Selective Ad Hoc On-Demand Multipath Distance Vector (LBS-AOMDV) algorithm is considered as baseline. Experiments taking place on SimuLTE (OMnet++) reveal that the NAR algorithm incurs in (approximately) $11 \%$ less data messages when compared to LBS-AOMDV. Furthermore, NAR also consumes (approximately) 35\% less energy, although NAR sends more data messages.
\end{abstract}

Resumo. Esse artigo descreve um algoritmo de roteamento para comunicação dispostivo-à-dispositivo (D2D) em arquiteturas celulares tendo como objetivo estender a cobertura das estações base em cenários de segurança pública. $O$ algoritmo proposto leva em consideração que as comunicações $D 2 D$ em redes celulares são gerenciadas pela estação base. Considera-se como baseline, o algoritmo Load Balancing Based Selective Ad hoc On-Demand Multipath Distance Vector (LBS-AOMDV). Os experimentos foram realizados no simulador SimuLTE (OMnet++) e os resultados demonstram que o algoritmo NAR perde aproximadamente $11 \%$ menos mensagens de dados quando comparado ao LBSAOMDV, apresentando um consumo de energia inferior em aproximadamente 35\%, mesmo com o algoritmo NAR enviando mais mensagens de dados em relação ao LBS-AOMDV.

\section{Introdução}

Para a próxima geração da telefonia móvel (5G), alguns requisitos como escalabilidade, resiliência e energia são necessários para uma arquitetura de comunicação prover uma comunicação que forneça qualidade de serviço ao usuário. No LTE ${ }^{1}$ Avançado (LTE-A) ${ }^{2}$, a comunicação D2D (Dispositivo para Dispositivo) é uma tecnologia candidata para satisfazer aos requisitos necessários e estender a cobertura da rede em cenários de segurança

\footnotetext{
${ }^{1}$ Long Term Evolution.

${ }^{2}$ LTE-A é um padrão de redes celulares que permite banda larga móvel com altas taxas de transmissão, possibilitando uma maior abrangência de comunicações de voz e transferência de dados.
} 
pública. Oportunidades significativas para criar e explorar alianças entre redes de segurança pública e redes comerciais LTE-A têm sido discutidas no $3 \mathrm{GPP}^{3}$ (3rd Generation Partnership Project) para permitir um aumento na capacidade agregada e uma melhora na capacidade da cobertura da rede [Lin et al. 2014]. Com a necessidade de reestruturação das arquiteturas de comunicação para atender aos novos requisitos, novas propostas de implementação e utilização de novas tecnologias têm surgido com o objetivo de diminuir custos operacionais, buscando uma arquitetura que seja mais dinâmica e com uma melhor eficiência energética [Agiwal et al. 2016].

A comunicação D2D em redes celulares é definida como uma comunicação direta entre dois dispositivos móveis sem passar pela estação base (BS) ou núcleo da rede. Comunicação D2D não é transparente para a rede celular e pode ocorrer dentro (Inband) ou fora (Outband) do espectro celular. Contudo, usuários móveis das redes celulares de hoje utilizam serviços que necessitam de altas taxas de dados que podem ser atingidos através de uma comunicação direta entre os dispositivos, tendo a comunicação D2D não limitada apenas a eficiência espectral, mas permitindo a potencialização no aumento da taxa de transferência, eficiência energética, diminuição dos atrasos e conformidade da rede em diferentes cenários para diferentes casos de uso [Asadi et al. 2014].

Segurança Pública engloba vários setores incluindo a polícia, bombeiros, organizações militares, entre outras organizações para criar um ambiente estável diante de ameaças naturais e/ou provocadas pelo homem. Em cenários como emergências em áreas urbanas, desastres naturais em áreas rurais, desastres nas fronteiras envolvendo diferentes nações e grandes eventos torna-se um desafio prover comunicação entre as diferentes organizações. Os cenários operacionais e as tecnologias utilizadas necessitam de requisitos operacionais como tempo de implantação, segurança, interoperabilidade, resiliência e taxa de dados para prover uma comunicação eficiente [Baldini et al. 2014].

Como principal contribuição, apresenta-se um algoritmo de roteamento (Roteamento Assistido pela Rede - NAR) para comunicação D2D em arquiteturas celulares que tem como objetivo estender a cobertura das estações base em cenários de segurança pública. Do ponto de vista de arquiteturas de comunicação D2D, diferentemente das redes Mobile ad hoc Network $\left(\mathrm{MANETs}^{4}\right.$ ) [Dow et al. 2005] e Cognitive Radio Networks $\left(\mathrm{CRN}^{5}\right)$ [Kamruzzaman et al. 2011], as comunicações D2D em redes celulares são gerenciadas pela estação base [Asadi et al. 2014]. Diferentemente de outros trabalhos, o algoritmo proposto leva em consideração a característica em que a definição das rotas para a comunicação D2D é definida pela estação base através do menor caminho e da melhor qualidade do canal, alocando blocos de recursos para os dispositivos móveis que participarão da comunicação.

$\mathrm{Na}$ avaliação do algoritmo, considera-se como baseline o algoritmo Load Balancing Based Selective Ad hoc On-Demand Multipath Distance Vector (LBS-AOMDV) [Tata and Kadoch 2014]. Os algoritmos foram implementados no simulador SimuLTE

\footnotetext{
33GPP é uma associação que reúne uma série de órgãos normativos de telecomunicações, abrangendo tecnologias de redes de telecomunicações celulares.

${ }^{4}$ MANETs são redes continuamente auto-configuráveis sem infraestrutura para conexão de dispositivos móveis sem fio.

${ }^{5} \mathrm{CRN}$ é uma tecnologia habilitada para suportar acesso dinâmico do espectro, considerada como uma das tecnologias mais promissoras para futuras comunicações sem fio.
} 
(OMnet++) ([Virdis et al. 2014]) e os resultados demonstram que o algoritmo NAR perde aproximadamente $11 \%$ a menos mensagens de dados em relação ao algoritmo LBSAOMDV e tem um consumo de energia aproximado de 35\% menor em relação ao algoritmo LBS-AOMDV, mesmo com o algoritmo NAR enviando mais mensagens de dados em relação ao algoritmo LBS-AOMDV.

Esse trabalho encontra-se organizado da seguinte forma. A seção 2 apresenta os trabalhos relacionados. A seção 3 apresenta o algoritmo NAR. A seção 4 apresenta o algoritmo utilizado como baseline. A seção 5 apresenta os experimentos e resultados encontrados. A seção 6 conclui o trabalho.

\section{Trabalhos Relacionados}

A comunicação D2D é uma nova tendência em redes celulares. [Asadi et al. 2014] categoriza os diversos tipos de comunicação D2D, distribuídas entre comunicação dentro da banda (Inband) e fora da banda (Outband). Comunicações dentro de banda usam o mesmo espectro para as comunicações D2D e celular. Já as comunicações fora de banda exploram enlaces fora do espectro celular com o objetivo de reduzir o risco de interferências. Em termos de contexto, esse trabalho propõe uma estratégia baseada em comunicação dentro de banda permitindo alto controle sobre o espectro celular.

Em [Tata and Kadoch 2014] é proposto uma solução para o roteamento de múltiplos caminhos em comunicação D2D sobre Redes Heterogêneas (HetNets) ${ }^{6}$, tendo como particularidade oferecer informações sobre a disponibilidade de largura de banda em cada rota e a redução no número de nós que recebem mensagens de requisição de rota.

Em [Melki et al. 2016] é proposto um modelo de alocação de recursos para habilitar comunicação multihop para comunicações D2D em redes celulares LTE-A. É formulado um problema de otimização de taxa de transferência sob as restrições de SINR (Signal-to-interference-plus-noise ratio) dos usuários móveis.

Em [Luz and Garcia 2015] foi proposto um algoritmo para implementar um mecanismo de tráfego adaptativo a partir de medidas que verificam a variação de SINR em estações com baixa mobilidade. A proposta é diminuir os quadros de gerenciamento e controle que tem como objetivo apenas a verificação contínua da conexão.

O algoritmo Interference Aware Routing Algorithm (IAR) [Yuan et al. 2014] foi proposto para reduzir a interferência D2D causada pela estação base, implementado num modelo baseado em geometria estocástica. O processo de roteamento D2D ocorre ao longo da borda da célula das estações base, em que a distância para estações base adjacentes é maximizada e a interferência agregada para as estações base são minimizadas.

Para minimizar o número de saltos e reduzir o consumo de energia, em [Ren et al. 2013] foi proposto um algoritmo de roteamento ciente de interferência. O algoritmo observa informações geométricas, restrições de interferência e restrições das taxas D2D com foco em reduzir sua complexidade computacional.

De forma geral, esse trabalho diferencia-se dos anteriores pela proposição de um algoritmo de roteamento que utiliza a estação base para gerenciar e definir as rotas a serem

\footnotetext{
${ }^{6}$ HetNets consistem em redes celulares clássicas que fornecem cobertura total para uma área ampla e células que fornecem capacidade extra para algumas demandas de tráfego [Spiess et al. 2014].
} 
utilizadas na comunicação D2D, analisando o caminho mais curto, realizando a análise da qualidade do canal e alocando os recursos para as rotas selecionadas.

\section{Algoritmo "Roteamento Assistido pela Rede - NAR"}

NAR é um algoritmo de roteamento fora da banda (de acordo com a classificação de [Asadi et al. 2014]), que visa estender a cobertura celular através da comunicação D2D ou fornecer cobertura temporária quando as estações base estão sobrecarregadas ou inoperantes. Disastres naturais em áreas rurais é um cenário típico em que a comunicação é um dos maiores desafios devido a falta de infraestrutura de comunicação ou porque estão degradadas ou destruídas (terremotos, enchentes). Desta forma, a comunicação D2D pode ser utilizada para estender a cobertura das estações base que estão próximas ao local do evento. A proposta do algoritmo presume as seguintes premissas: a) roteamento D2D é definido pela estação base; b) a comunicação D2D ocorre no espectro celular (InbandUnderlay) e c) cada rota tem recursos que são alocados para a comunicação D2D.

NAR define múltiplas rotas entre a estação base e os dispositivos móveis, de acordo com o caminho mais curto e com o melhor indicador de qualidade do canal $\left(\mathrm{CQI}^{7}\right)$, abaixo de um limiar mínimo ${ }^{8}$. O algoritmo NAR é iniciado quando a estação base (BS) recebe uma solicitação ( $R R O U T E$ ) de um dispositivo móvel (UE) para realizar uma comunicação D2D. Portanto, a BS estabelece uma rota para conectar-se ao UE. O algoritmo Dijkstra é aplicado para encontrar o menor caminho entre a BS e o UE. Um pacote de solicitação $(S R E Q)$ é enviado da BS para o UE, através do caminho retornado pelo algoritmo Dijkstra, solicitando para o UE o conjunto de vizinhos (filhos) e a qualidade do canal de cada vizinho.

Antes de responder com um pacote de resposta $(S R E P)$, o UE realiza um broadcast com uma mensagem de "Olá" (Hello - msg) com um tempo de vida (TTL) igual a 1. Todos os vizinhos do UE, respondem com um pacote de resposta "Olá" (Hello - rep) informando o UE sobre sua existência e sobre a qualidade do seu canal. O conjunto de filhos e os CQIs são transmitidos para a BS através de um pacote de resposta $S R E P$. Da mesma forma, (Hello - msg) e (Hello - rep) são trocados com a BS para ter o conjunto de filhos e os CQIs disponíveis.

Para garantir as múltiplas rotas livres de laços, cada dispositivo envia um $R R E Q$ para definir seu atributo $R R E Q_{T} x$ como desligado. Além disso, cada um recebendo o pacote deve colocar o atributo $R R E Q_{R} x$ como desligado. Uma exceção é feita para a $\mathrm{BS}$, que não define o $R R E Q_{T} x$ para desligado até enviar o $R R E Q$ para todos os seus filhos escolhidos como membro das múltiplas rotas. Da mesma maneira, o UE não define seu $R R E Q_{R} x$ para desligado, uma vez que não recebeu $R R E Q$ de todos os seus filhos escolhidos como membro das múltiplas rotas.

Contudo, a BS tem que enviar ( $R R E Q)$ somente para os seus filhos. Sendo que, cada dispositivo tem que selecionar o melhor filho a qual o $R R E Q$ é encaminhado. Obviamente, o destino não envia o $R R E Q$ para qualquer lugar, mas pode receber de mais de um filho, estabelecendo múltiplos caminhos. É por isso, que o $R R E Q_{T} x$ do destino

\footnotetext{
${ }^{7} \mathrm{CQI}$ significa Indicador de Qualidade de Canal. É um indicador que carrega informações sobre a qualidade do canal de comunicação.

${ }^{8}$ Nós consideramos os 15 valores de CQI apresentados no release 8 do LTE-A avaliado em http: //www.3gpp.org/specifications/releases/72-release-8.
} 
UE não está definido até receber uma solicitação de rota de todos os filhos escolhidos como membro de uma rota. NAR é apresentado no Algoritmo 1.

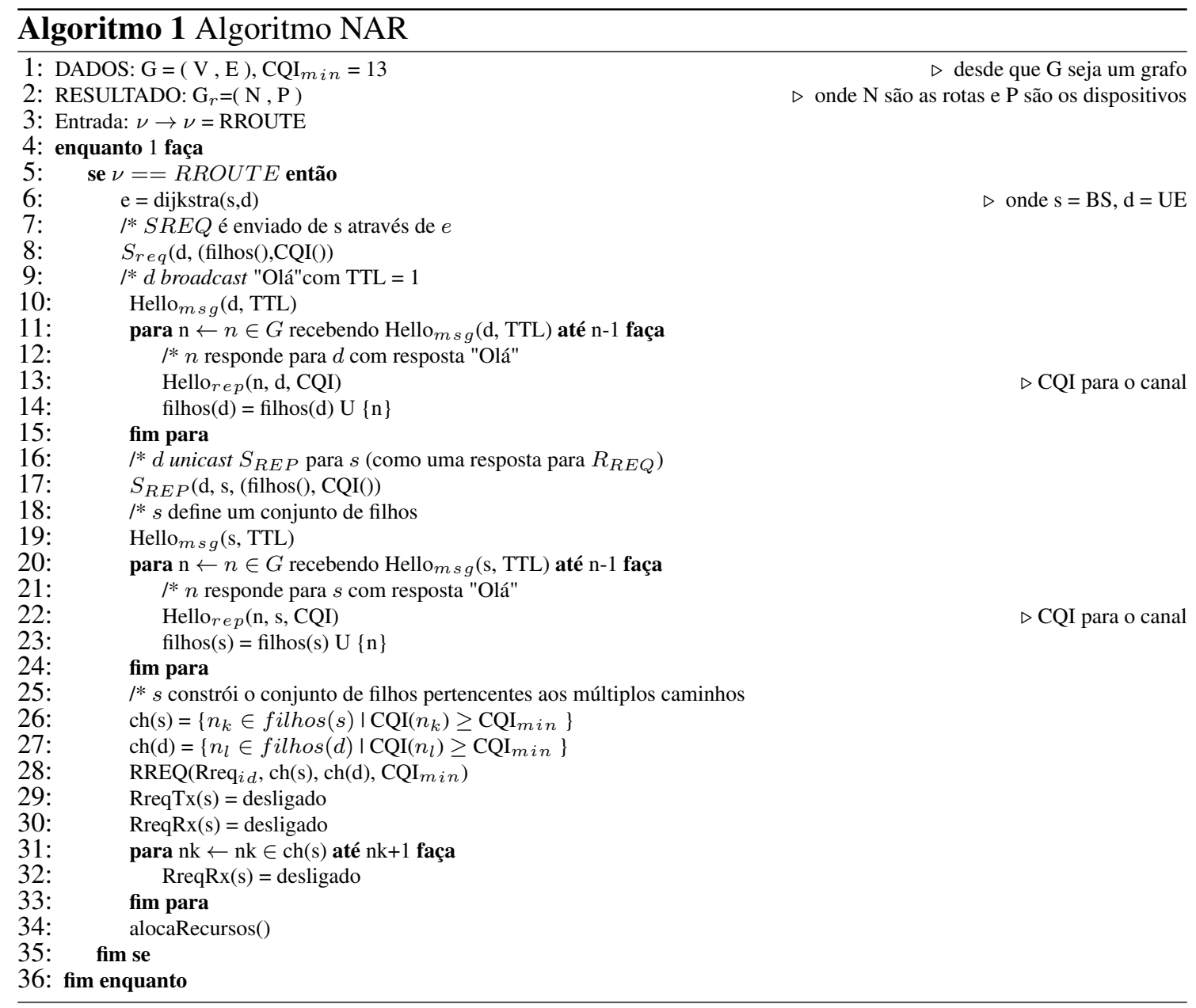

Assim que o UE recebe o $R R E Q$, ele responde com uma resposta de rota $R R E P$. $R R E P$ adiciona informações simples sobre o CQI do canal, permitindo escolher rotas para os múltiplos caminhos. Após a definição dos múltiplos caminhos, é realizado o procedimento de alocação de recursos, apresentado no Algoritmo 2, em que cada solicitação de rota (RROUTE) de um UE, é definido duas rotas, com blocos de recursos distintos, sendo uma rota para a comunicação D2D e a outra rota servindo de auxiliar em caso de falha. Para o encerramento da rota, foi definido um tempo de vida da rota igual a $60 \mathrm{~s}$.

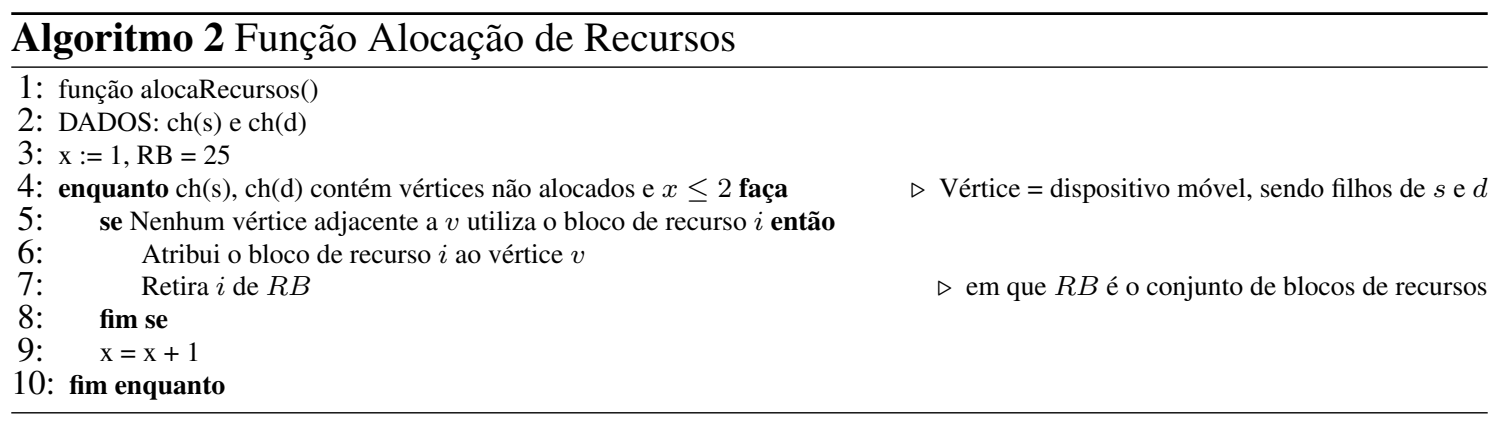

Dado o funcionamento do algoritmo, podemos rotular o algoritmo como pró-ativo 
ao selecionar mais de uma rota para a comunicação D2D e reativo ao selecionar as rotas somente após uma requisição de rota (RROUTE).

\section{Baseline}

Compara-se nosso algoritmo com o algoritmo Load Balancing Based Selective Ad hoc On-Demand Multipath Distance Vector (LBS-AOMDV) que é um aprimoramento do algoritmo Ad hoc On-Demand Multipath Distance Vector (AOMDV). Ele é aplicado para definir múltiplos caminhos para compor as comunicações D2D para Segurança Pública sobre Redes Heterogêneas. Uma particularidade de LBS-AOMDV é oferecer informações sobre a largura de banda disponível de cada rota dentro dos múltiplos caminhos. Além disso, reduz o tráfego de controle, diminuindo o número de nós que recebem as solicitações $R R E Q$. Isto é viável uma vez que os remetentes das solicitações $R R E Q$ selecionam os dispositivos que podem receber os pacotes.

$\mathrm{O}$ algoritmo LBS-AOMD encaminha $R R E Q$ da origem para localizar o destino. Mas, ao contrário do algoritmo AOMDV, o dispositivo remetente não inunda os pacotes através de toda a rede. Em vez disso, para cada transmissão, ele escolhe o melhor entre seus filhos para ser o receptor $R R E Q$.

\section{Experimentos e Resultados}

Para realizar uma abordagem científica no planejamento experimental, foram definidos os parâmetros da simulação e a forma da coleta dos dados, para posteriormente realizar a análise dos dados, refutando ou corroborando o algoritmo proposto. A seguir uma descrição para cada fase realizada do planejamento experimental.

No Planejamento Pré-Experimental, são definidos os fatores de interesse do algoritmo NAR, os níveis dos fatores e as variáveis de respostas, descritas como: a) Reconhecimento e definição do problema: Garantir a confiabilidade na entrega dos pacotes, minimizando a perda de pacotes sem inviabilizar o tempo da rede; b) Escolha de fatores de interesse, níveis e intervalos: $\mathrm{Na}$ tabela 1 foram definidos os parâmetros utilizados nas simulações; c) Seleção da variável de resposta: mensagens de dados enviadas, mensagem de dados recebidas, nível do indicador de qualidade do canal (CQI) e energia consumida. Todas as variáveis foram analisadas para cada algoritmo avaliado, exceto para o algoritmo LBS-AOMDV em que o CQI não está disponível.

\begin{tabular}{|l|l|}
\hline Parâmetros & Valor \\
\hline Frequência da Portadora & $2 \mathrm{GHz}$ \\
Largura de Banda & $5 \mathrm{MHz}$ (25 RBs) \\
Modelo de Perda & ITU Urban Macro \\
Modelo de Desvanecimento & Jakes \\
Potência dispositivo móvel Tx & $26 \mathrm{~dB}$ \\
Ruído & $5 \mathrm{~dB}$ \\
Perda no Cabo & $2 \mathrm{~dB}$ \\
Tempo de Simulação & $3600 \mathrm{~s}$ \\
\hline
\end{tabular}

Tabela 1. Parâmetros da Simulação no SimuLTE ([Virdis et al. 2014]).

O Planejamento de Experimentos, foi baseado nas definições do planejamento Pré-Experimental, sendo definidos os experimentos e variáveis de respostas: a) Fatores de interesse: mensagens de dados enviadas, mensagens de dados recebidas, nível do CQI e energia consumida; b) Hipótese Nula (H0): H0: $T 1=T 2=0$; c) H1 (unilateral / 
bilateral): $H 1: T i \neq 0 \rightarrow$ (Hipótese Alternativa) $\rightarrow$ para no mínimo um $i$; d) Nível de significância:: $\alpha=0.05$. Nesse contexto, $T 1$ e $T 2$ representam os algoritmos NAR e LBS-AOMDV e $\alpha$ a confiabilidade dos dados. A análise dos dados foram realizadas através de uma ferramenta de desenvolvimento estatístico denominada $\mathrm{R}^{9}$.

Para a coleta dos dados, foram realizadas simulações no simulador SimuLTE e executadas de acordo com os parâmetros descritos na tabela 1. A aleatorização dos cenários foi utilizada para evitar problemas computacionais. Cada experimento foi realizado 33 vezes, com duração de 3600 segundos para cada simulação.

$\mathrm{Na}$ fase de Análise dos Experimentos, o propósito foi validar e comparar o algoritmo com seu baseline. Para a validação do algoritmo, foram verificados se os procedimentos definidos para o algoritmo estavam funcionando corretamente. Para essa validação, foram definidos três cenários distintos: com uma estação base e cinco dispositivos móveis, duas estações base e cinco dispositivos móveis e três estações base e cinco dispositivos móveis. A figura 1 representa o cenário com 3 estações base, em que foram avaliados o comportamento do algoritmo com relação a definição da rota, ao envio de dados entre os dispositivos móveis e o encerramento da comunicação. Nesses experimentos os dispositivos móveis se comportam de forma estacionária.

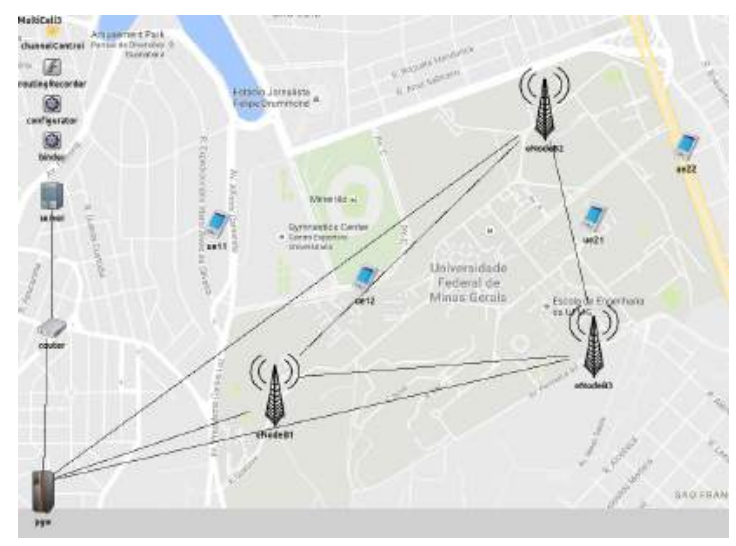

Figura 1. Cenários para validação do funcionamento do algoritmo NAR.

Para a comparação do algoritmo NAR com o seu baseline, o objetivo foi analisar o funcionamento do algoritmo em relação as métricas de desempenho. Foram realizados 33 experimentos com o algoritmo NAR e 33 experimentos com o algoritmo LBSAOMDV em um cenário com uma estação base e 100 dispositivos móveis dispostos de forma estacionária e aleatória dentro da área de cobertura celular $(800 \mathrm{~m}$ x 800m), em que os dispositivos móveis realizam comunicações celulares de forma aleatória e simultânea com a comunicação D2D, sendo que o dispositivo móvel alocado para formar uma rota de comunicação D2D não pode realizar comunicação celular simultaneamente.

Para esse experimento, a figura 2(a) apresenta a média de mensagens de dados enviadas durante as simulações referente ao algoritmo NAR e o algoritmo LBS-AOMDV, demonstrando que o algoritmo NAR envia aproximadamente $11 \%$ a mais de mensagens

\footnotetext{
${ }^{9} \mathrm{R}$ é uma linguagem e também um ambiente de desenvolvimento integrado para cálculos estatísticos e gráficos https: / / www.r-project.org/
} 
para o cenário determinado. A figura 2(b) apresenta a média das $\%$ de mensagens que são entregues e a média da \% de mensagens de dados perdidas durante as simulações, demonstrando a eficiência do algoritmo NAR na entrega de mensagens em comparação com o algoritmo LBS-AOMDV, sendo que o NAR tem uma taxa de perda de mensagens de aproximadamente $4,73 \%$ e o LBS-AOMDV uma taxa de perda aproximada de $15,4 \%$.

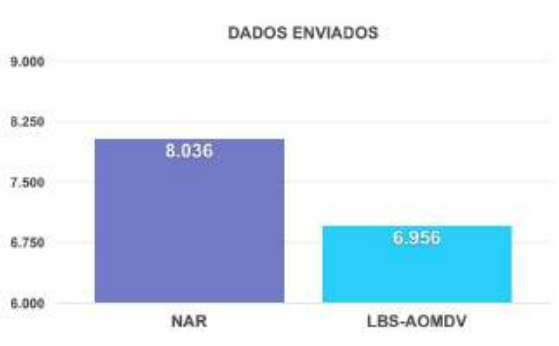

(a) Mensagens enviadas do algoritmo NAR e LBS-AOMDV.

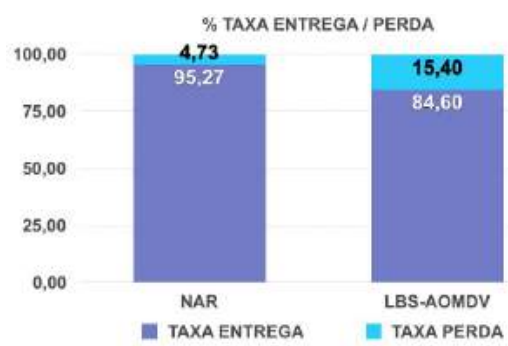

(b) Algoritmo NAR X LBS-AOMDV com relação a \% de entrega e perda de mensagens de dados.

Figura 2. Mensagens enviadas do algoritmo NAR X LBS-AOMDV.

Na figura 3, é representado o CQI, que determinada a qualidade do canal das comunicações para o algoritmo NAR em relação a distância (metros) entre os dispositivos móveis, em que a camada Radio Resource Control (RRC) da estação base, baseada nas informações enviadas pelo dispositivo móvel, determina algumas decisões com base em medições do nível de sinal das células. Além dessa função, essa camada envia mensagens de broadcast contendo informações do sistema e controla as medições dos parâmetros dos dispositivos móveis como a periodicidade do CQI. Quanto maior for o valor do CQI, melhor é a qualidade do canal.

Analisando a figura 3, dado uma distância em que $R<50 \mathrm{~m}$, podemos obter uma boa qualidade do canal, preservando a qualidade de transferência no canal de comunicação D2D. Os valores de CQI x Distância referente a figura 3, foram obtidos nas simulações de validação do algoritmo NAR, conforme figura 1.

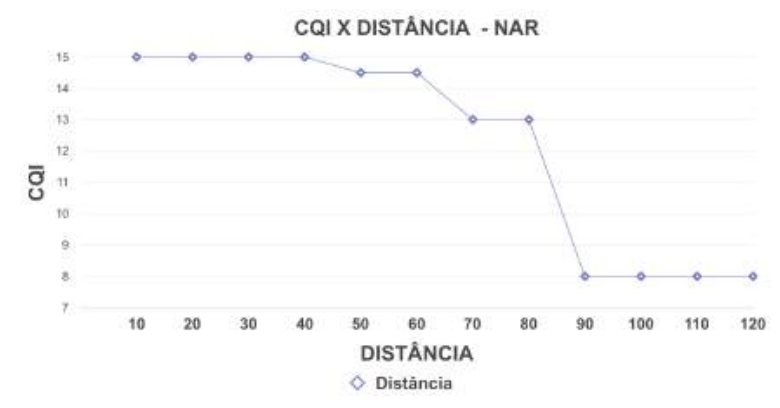

Figura 3. Indicador de qualidade do canal para o algoritmo NAR.

Para a avaliação do consumo energético nos dispositivos móveis, foram definidos alguns parâmetros para avaliar o consumo de energia na comunicação D2D, como por exemplo, a potência consumida para transmissão de pacotes de dados e roteamento. A potência consumida para recepção de pacotes e pacotes de controle não foram contabilizados nas simulações. A tabela 2, apresenta algumas informações sobre o total de pacotes enviados e a energia consumida pelos dispositivos móveis nas simulações. 


\begin{tabular}{|l|l|l|}
\hline Descrição & NAR & LBS \\
\hline Energia Inicial $(\mathrm{J})$ & 10000 & 10000 \\
Pacotes Dados & 8036 & 6956 \\
Pacotes Roteamento & 1603 & 2207 \\
Total Pacotes & 9636 & 9163 \\
Potência consumida para transmissão $(\mathrm{mW})$ & 660 & 660 \\
Tempo de Simulação & $3600 \mathrm{~s}$ & $3600 \mathrm{~s}$ \\
Energia Total Consumida & 2455 & 3324 \\
\hline
\end{tabular}

Tabela 2. Pacotes e Energia Consumida.

A figura 4 e a tabela 2, demonstra que o algoritmo NAR perde aproximadamente $11 \%$ a menos mensagens de dados em relação ao LBS-AOMDV e tem um consumo de energia aproximado de $35 \%$ menor em relação ao algoritmo LBS-AOMDV, mesmo com o algoritmo NAR enviando mais mensagens de dados.

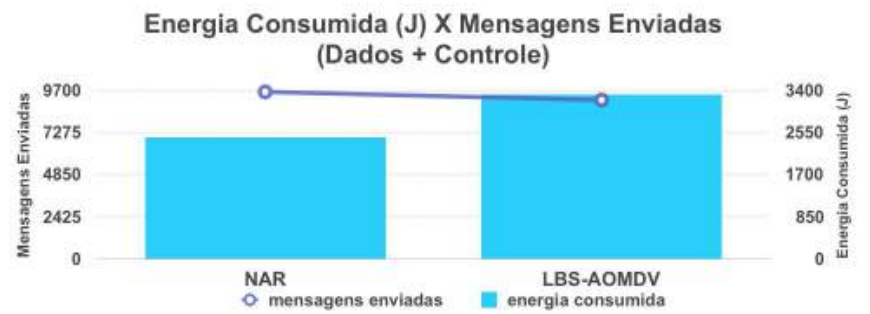

Figura 4. Mensagens Enviadas x Energia Consumida.

Através dos experimentos realizados na comparação do algoritmo NAR em relação ao algoritmo LBS-AOMDV, foi possível verificar que a abordagem proposta para o roteamento, tendo como premissa uma participação efetiva da estação base na determinação da rota, a escolha da rota baseado no CQI do canal e a alocação de blocos de recursos para cada rota, permite definir uma rota com menor interferência e melhor entrega das mensagens, contribuindo para uma diminuição no consumo energético dos dispositivos móveis, refutando a Hipótese Nula com um nível de significância de 95\%.

\section{Conclusão}

Nesse trabalho foi proposto o algoritmo de roteamento definido como Roteamento Assistido pela Rede (NAR) que se enquadra na categoria de Inband D2D - Underlay, tendo como uma das premissas o gerenciamento da rota definido pela estação base, em que as métricas de menor caminho, qualidade do canal e a alocação de blocos de recursos são utilizados com o propósito de diminuir a interferência entre as comunicações e melhorar a utilização do espectro celular. Os experimentos foram realizados no SimuLTE e o algoritmo NAR comparado com o algoritmo LBS-AOMDV. Os resultados demonstram que o algoritmo NAR perde aproximadamente $11 \%$ a menos mensagens de dados em relação ao LBS-AOMDV e tem um consumo de energia aproximado de 35\% menor em relação ao algoritmo LBS-AOMDV, mesmo com o algoritmo NAR enviando mais mensagens de dados, atendendo aos requisitos para aplicações de Segurança Pública. Como trabalhos futuros, será analisado a quantidade de rotas ideal para cada solicitação, de forma a garantir melhor resiliência, realizar experimentos com os dispositivos em movimento e analisar o comportamento do algoritmo em redes reais.

\section{Agradecimentos}

O presente trabalho foi realizado com o apoio financeiro da CAPES - Brasil. 


\section{Referências}

Agiwal, M., Roy, A., and Saxena, N. (2016). Next generation 5G wireless networks: A comprehensive survey. IEEE Communications Surveys and Tutorials, 18(3):16171655.

Asadi, A., Wang, Q., and Mancuso, V. (2014). A survey on device-to-device communication in cellular networks. IEEE Communications Surveys and Tutorials, 16(4):18011819.

Baldini, G., Karanasios, S., Allen, D., and Vergari, F. (2014). Survey of Wireless Communication Technologies for Public Safety. Communications Surveys Tutorials, IEEE, 16(2):619-641.

Dow, C. R., Lin, P. J., Chen, S. C., Lin, J. H., and Hwang, S. F. (2005). A Study of Recent Research Trends and Experimental Guidelines in Mobile Ad Hoc Networks. 19th International Conference on Advanced Information Networking and Applications (AINA'05) Volume 1 (AINA papers), 1:72-77.

Kamruzzaman, S. M., Kim, E., and Jeong, D. G. (2011). Spectrum and Energy Aware Routing Protocol for Cognitive Radio Ad Hoc Networks. Communications Society, pages 344-349.

Lin, X., Andrews, J. G., Ghosh, A., and Ratasuk, R. (2014). An overview of 3GPP device-to-device proximity services. IEEE Communications Magazine, 52(4):40-48.

Luz, K. S. and Garcia, H. (2015). Caracterização da Micromobilidade em Redes Sem Fio Infraestruturadas pela Variação da Relação Sinal-Ruído. 7o SBCUP - Simpósio Brasileiro de Computação Ubíqua e Pervasiva - SBCUP/CSBC, pages 131-140.

Melki, L., Najeh, S., and Besbes, H. (2016). Interference Management Scheme for Network-Assisted Multi-Hop D2D Communications. IEEE 27th Annual International Symposium on Personal, Indoor and Mobile Radio Communications (PIMRC), pages $1-5$.

Ren, P., Du, Q., and Sun, L. (2013). Interference-aware routing for hop-count minimization in wireless D2D networks. 2013 IEEE/CIC International Conference on Communications in China - Workshops, CIC/ICCC 2013, pages 65-70.

Spiess, J., Joens, Y. T., Dragnea, R., and Spencer, P. (2014). Using Big Data to Improve Customer Experience and Business Performance. Bell Labs Technical Journal, 18(4):3-17.

Tata, C. and Kadoch, M. (2014). Multipath routing algorithm for device-to-device communications for public safety over LTE Heterogeneous Networks. 2014 1st International Conference on Information and Communication Technologies for Disaster Management, ICT-DM 2014.

Virdis, A., Stea, G., and Nardini, G. (2014). SimuLTE - A Modular System-level Simulator for LTE / LTE-A Networks based on OMNeT ++. Proceedings of SimulTech, pages $28-30$.

Yuan, H., Guo, W., and Wang, S. (2014). Emergency route selection for D2D cellular communications during an urban terrorist attack. 2014 IEEE International Conference on Communications Workshops, ICC 2014, pages 237-242. 